\title{
Experimentos a alta presión y alta temperatura: Ventanas al universo
}

\author{
D. SANTAMARÍA-PÉREZ \\ Departamento de Química-Física I, Universidad Complutense de Madrid, Madrid, España. \\ dsantamaria@quim.ucm.es
}

Recibido: $15 / 03 / 2011$

Aceptado: 27/04/2011

\section{Resumen}

A partir de los indicios sobre la composición terrestre que proporcionan los métodos indirectos, como la propagación de ondas sísmicas, es posible recrear en el laboratorio condiciones de presión y temperatura similares a las del interior de la Tierra y de otros planetas, y estudiar cómo afectan estas condiciones a un metal o mineral determinado. Estos experimentos son, por tanto, ventanas al universo. El objetivo de este capítulo es mostrar la enorme potencia de las técnicas experimentales de alta presión y alta temperatura y dar una visión de conjunto de su aplicación en distintos ambitos de la geofísica. Además, presentaremos al consorcio MALTA-Consolider, que agrupa a la mayor parte de los laboratorios españoles dedicados a la alta presión, y las instalaciones experimentales de que disponen.

Palabras clave: Técnicas experimentales, alta presión, alta temperatura, celdas de diamante, calentamiento láser, MALTA-Consolider.

\section{High-pressure high-temperature experiments: Windows to the Universe}

\begin{abstract}
From Earth compositional arguments suggested by indirect methods, such as the propagation of seismic waves, is possible to generate in the laboratory pressure and temperature conditions similar to those of the Earth or other planet interiors and to study how these conditions affect to a certain metal or mineral. These experiments are, therefore, windows to the Universe. The aim of this chapter is to illustrate the huge power of the experimental high-pressure high-temperature techniques and give a global overview of their application to different geophysical fields. Finally, we will introduce the MALTA Consolider Team, which gather most of the Spanish high-pressure community, and present their available high-pressure facilities.

Keywords: Experimental techniques, high-pressure, high-temperature, diamond-anvil cell, laser heating, MALTA-Consolider.

Sumario: Introducción. 1. Técnicas de alta presión (AP) y alta temperatura (AT). 1.1. Alta presión dinámica. 1.2. Alta presión estática. 2. Técnicas de caracterización a alta presión y a alta temperatura. 2.1. Radiación sincrotrón. 2.2. Difracción de rayos X. 2.3. Dispersión inelástica de rayos X. 3. Consorcio español de altas presiones: MALTA-Consolider Team. 4. Agradecimientos. Referencias bibliográficas.
\end{abstract}

Referencia normalizada

Santamaría-Pérez, D., (2011). Experimentos a alta presión y alta temperatura: Ventanas al universo. Física de la Tierra Vol. 23 Núm. 1 (2011): 11-27

En memoria al Prof. Fiedrich Liebau

Berlin, 31/05/1926 - Kiel, 11/03/2011 


\section{Introducción}

Desde siempre, el hombre ha anhelado conocer la estructura íntima de la Tierra; sin embargo, su estudio es una tarea compleja ya que el interior de nuestro planeta es en gran medida inaccesible a través de medidas directas. Únicamente una pequeñisima parte de su material constituyente puede ser extraida y analizada. Nuestro conocimiento proviene pues del análisis de medidas indirectas. Así, por ejemplo, la interpretación de los datos obtenidos a partir de la propagación de las ondas sísmicas nos da una información muy importante de la densidad y la isotropía de los materiales que forman nuestro planeta. El estudio de la velocidad de estas ondas ha permitido determinar varias discontinuidades sísmicas y plantear un modelo geológico basado en una división en capas de la estructura terrestre (Dziewonski \& Anderson, 1981, Jordan, 1979) (figura 1). Según este modelo geostático la Tierra está dividida en las siguientes capas:

- La capa sólida más externa, denominada corteza, con un espesor que varía de 10 y $70 \mathrm{Km}$.

- Una capa intermedia sólida, llamada manto, que llega hasta una profundidad de unos $2900 \mathrm{Km}$ y es de naturaleza no metálica. Ésta región se subdivide a su vez, principalmente, en manto superior, zona de transición y manto inferior.

- Un núcleo metálico que se divide en dos regiones: una externa fundida y otra más interna sólida.

Otros métodos indirectos de estudio, como son la composición de los meteoritos o la magnitud de los campos magnético y gravitatorio, entre otros, nos permiten inferir la dinámica interna y los perfiles de densidad, ya no solo de la Tierra, sino de otros planetas y satélites del Sistema Solar.

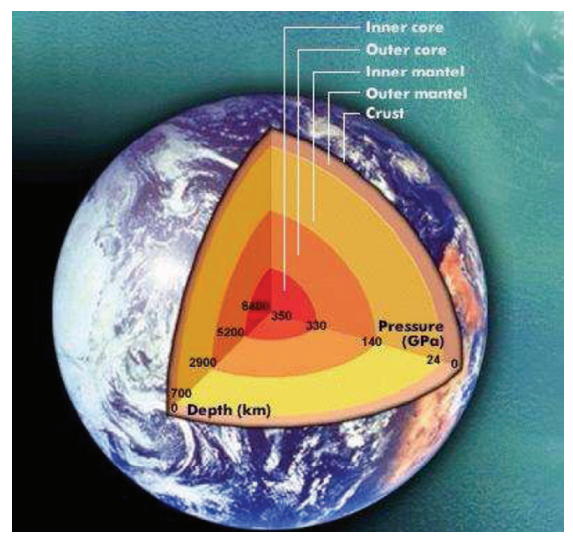

Fig. 1. Representación de las distintas capas existentes en el interior de la Tierra donde se muestra la variación de presión con la profundidad.

Imagen:http://msnbcmedia4.msn.com/j/msnbc/Components/Photos/070920/070920_earth_i nside_hmed_02.grid-6x2.jpg 
A partir de los indicios sobre la composición terrestre que proporcionan los métodos que acabamos de mencionar, es posible recrear en el laboratorio condiciones de presión y temperatura similares a las del interior de la Tierra y de otros planetas, y analizar así cómo afectan a un metal o mineral determinado. Estos experimentos son, por tanto, como reza el título del capítulo, ventanas a traves de las cuales podemos observar los procesos que acontecen en las profundidades de los planetas, allí donde nunca podremos llegar. En los últimos 40 años se ha producido un rápido desarrollo tanto teórico como experimental en el campo de la física de la materia condensada a alta presión. Estos progresos y los avances recientes en experimentos a alta y baja temperatura nos han permitido estudiar experimentalemente el comportamiento de distintos materiales en un rango de presiones-temperaturas (P-T) muy extenso. Los estudios experimentales suelen complementarse con cálculos teóricos que ayudan a comprender los resultados obtenidos y, en algunos casos, pueden llegar a predecir el comportamiento de la materia bajo ciertas condiciones. El objetivo de este capítulo es dar una breve introducción de los distintos métodos experimentales empleados para reproducir las condiciones extremas de presión y temperatura de las zonas de interés, asi como de las técnicas de caracterización estructural de la materia en estas condiciones. Conjuntamente, destacaremos varios estudios de especial relevancia en Geofísica que han usado este tipo de técnicas.

\section{Técnicas de alta presión (AP) y alta temperatura (AT)}

Comencemos diciendo que, en general, hay que considerar que la máxima presión que se puede alcanzar en un determinado experimento depende del volumen de la muestra que queramos estudiar, pues la energía para alcanzar una determinada presión escala directamente con el volumen considerado $($ Presión $=$ Fuer$\mathrm{za} /$ Superficie $=$ Energía/Volumen). Es decir, conseguir grandes presiones requiere cantidades de muestra diminutas. Por otra parte, siempre hay que considerar la resistencia mecánica y térmica de los materiales empleados en los dispositivos de AP y AT y ser conscientes de que hay experiencias dificiles de llevar a cabo en un laboratorio.

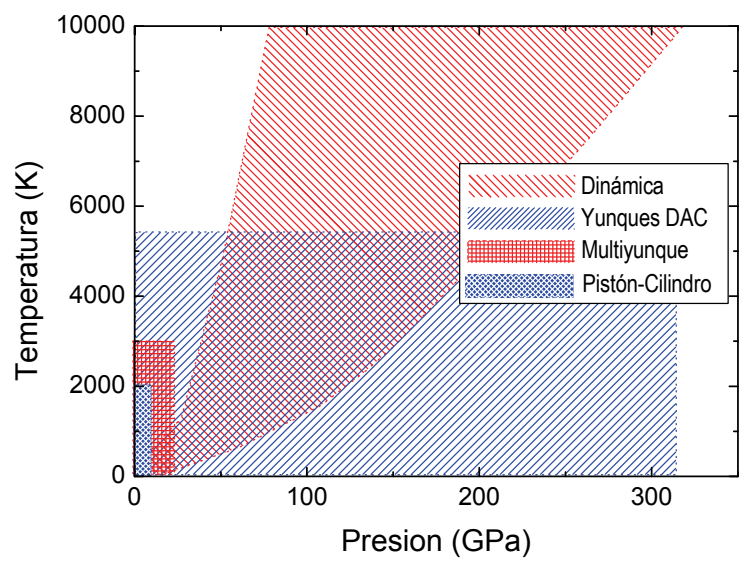

Fig. 2. Intervalos de presión y temperatura accesibles con las distintas técnicas de alta presión y alta temperatura. 
Generalmente, las técnicas de alta presión se pueden clasificar según su caracter dinámico o estático. En la figura 2 se representa el rango de presiones y temperaturas accesibles con las distintas técnicas experimentales existentes.

\subsection{Alta presión dinámica}

En las técnicas de alta presión dinámica la materia se comprime por medio del impacto de proyectiles a velocidad supersónica (ver figura 3 ) o láseres pulsados de gran potencia. De esta manera se crean ondas de choque que generan condiciones extremas de presión y temperatura de forma simultánea en la muestra a estudiar (hasta varios cientos de GPa y varios miles de grados) durante periodos de tiempo extremadamente cortos (del orden del microsegundo). Por esta razón se requieren técnicas de caracterización electrónica, óptica y estructural muy rápidas (Nellis, 2006).

El proceso termodinámico en el frente de onda se considera adiabático y la temperatura aumenta como consecuencia de la compresión del material. Las discontinuidades encontradas en medidas de la velocidad de propagación de las ondas se deben a transiciones de fase en nuestro especimen. Las temperaturas pueden ser determinadas mediante medidas cuidadosas de la radiación emitida por la muestra pero, debido a la extrema complejidad de estos experimentos, normalmente se suelen calcular a partir de resultados experimentales del calor específico y el parámetro de Grüneisen. Es importante recalcar que los resultados experimentales obtenidos mediante técnicas dinámicas han resultado determinantes para el progreso de la alta presión, pues el empleo de la relacción Rankine-Hugoniot ha permitido construir las ecuaciones de estado de referencia para los experimentos estáticos.

(a)

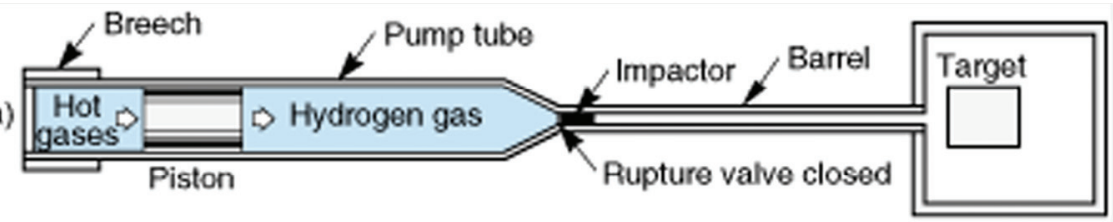

(b)

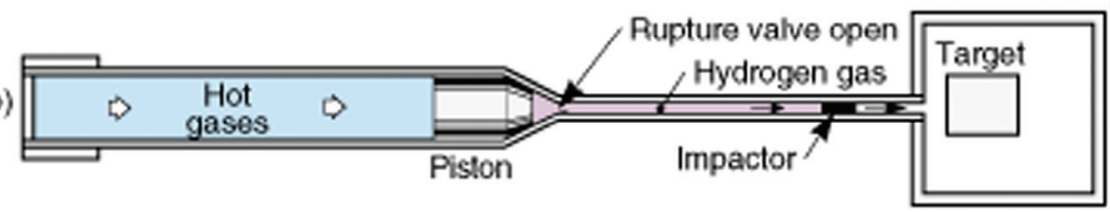

Fig. 3. Pistola de gas empleada para generar ondas de choque en la muestra mediante el impacto de un proyectil a altísima velocidad. (a) En una primera etapa, a partir de un explosivo, se generan gases extremadamente calientes que mueven un pistón comprimiendo el gas de hidrógeno. (b) En una segunda etapa, el gas a alta presión rompe la válvula. Esto provoca que el proyectil acelere en el cañon que le dirige a la muestra objetivo. Imagen: https://www.llnl.gov/str/Nellis.html 
Como se acaba de mencionar, esta técnica ha contribuido a entender el comportamiento de la materia en condiciones extremas. He aquí un claro ejemplo: La metalización del hidrógeno en Jupiter. Ya en 1935, Wigner y Huntington predijeron que el hidrógeno podría sufrir una transición de fase aislante-metal a altas presiones (Wigner \& Huntington, 1935). La primera evidencia de que el hidrógeno en Jupiter debía estar en un estado metálico fue proporcionada por las sondas Voyager en 1979. Solo una capa de hidrógeno metálico líquido podría originar el potentísimo campo magnético del planeta. Pues bien, recientemente, Nellis y colaboradores (Nellis et al., 1996, Weir et al., 1996) haciendo uso de las ondas de choque han podido probar la existencia de hidrógeno líquido con propiedades metálicas a presiones y temperaturas que corresponderían a las del interior de Jupiter (ver figura 4).

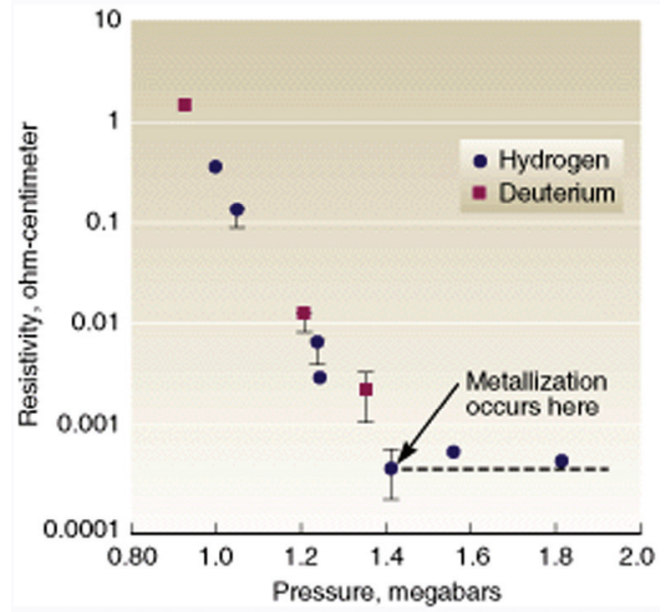

Fig. 4. De los experimentos de ondas de choque se deduce que la resistividad eléctrica del hidrógeno molecular decae drásticamente al aumentar la presión hasta valores propios de un metal (Nellis et al., 1996). Imagen: https://www.llnl.gov/str/Nellis.html

\subsection{Alta presión estática}

En este caso la muestra está contenida en una celda de alta presión. Dentro de los medios para generar presiones estáticas podemos diferenciar dos grandes grupos: las celdas de gran volumen (Large-volume Anvil Cells, LACs) y las celdas de yunque de diamante (Diamond Anvil Cells, DACs). Las celdas pistón-cilindro (permiten trabajar hasta unos $8 \mathrm{GPa}$ y $2000 \mathrm{~K}$ ), Paris-Edimburgo y multiyunque (hasta unos $24 \mathrm{GPa}$ y $2500 \mathrm{~K}$ ) pertenecen al primer grupo y pueden comprimir varios $\mathrm{mm}^{3}$ de muestra. El uso combinado de estás celdas y radiación monocromática sincrotrón permite obtener información in situ de las estructuras tanto cristalina como electrónica de las muestras (técnicas de caracterización tales como difracción de rayos $\mathrm{X}$, difracción de neutrones y espectroscopía de absorción de rayos X). La 
interacción de la radiación sincrotrón con la materia será brevemente tratada en el apartado 2.1 .

En las celdas de yunque de diamante (figura 5) se comprimen muestras muy pequeñas (del orden de $10^{-5} \mathrm{~mm}^{3}$ ) entre las puntas planas de dos diamantes enfrentados. En esta técnica se pueden alcanzar presiones muy altas (superiores a $250 \mathrm{GPa}$ ) gracias a la pequeña superficie plana de los diamantes, con un diámetro que oscila normalmente oscila entre 500 y 50 micras (la fuerza aplicada por unidad de area es muy elevada). La muestra queda alojada en el interior de una cavidad horadada en una junta metálica que confinará la muestra (ver figura 5). La preparación inicial de la junta (marcado) y el diámetro y la altura del orificio que servirá de cámara de presión dependerá de la presión máxima que se quiere alcanzar, del tamaño de los diamantes y de otros factores. Es también importante subrayar que el uso de un fluido transmisor de presión proporciona un medio quasi-hidrostático en un amplio rango de presiones. En la literatura podemos encontrar varios artículos monográficos que discuten en profundidad tanto el manejo como las aplicaciones de este tipo de celdas (Jayaraman, 1983, Eremets, 1996). El diamante fue elegido por su excepcional dureza y por sus propiedades ópticas, que permiten la observación in situ de nuevos fenómenos a alta presión. Además de las técnicas de caracterización mencionadas anteriormente para las LACs, en estas celdas es posible realizar medidas de absorción óptica y de espectróscopia Raman, Brillouin o Mossbauer.
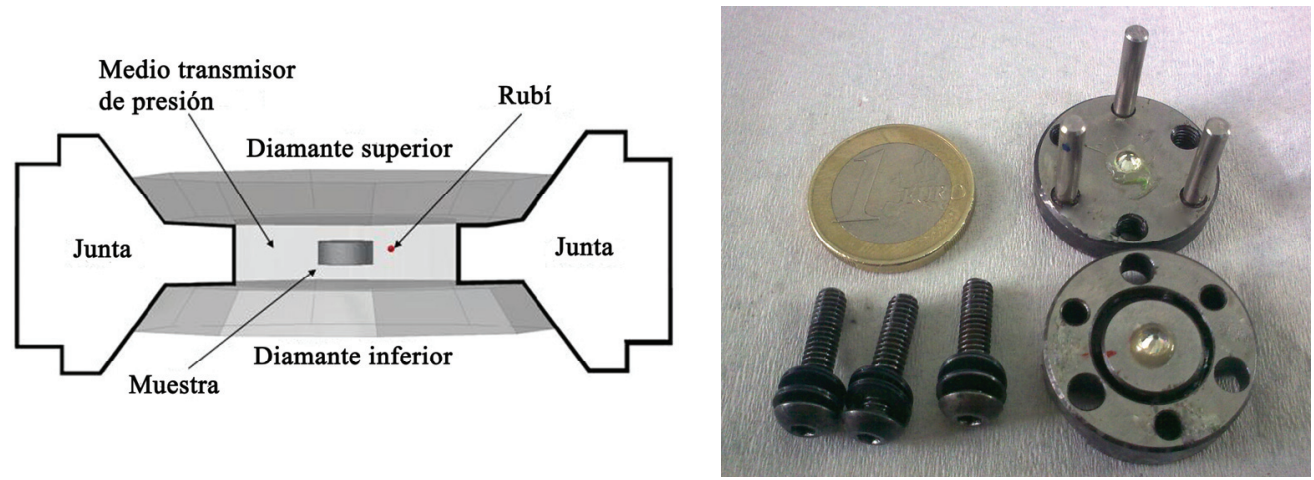

Fig. 5. (a) Esquema de la cámara de presión en una celda de yunques de diamante (DAC). La cavidad en la junta metálica confina la muestra, el medio transmisor de presión y el medidor de presión (rubí). (b) Foto de una DAC del tipo Merrill-Bassett modificada.

Una de las mayores dificultades en los experimentos a alta presión es determinar ésta de forma precisa y reproducible. Con este fín se emplean escalas de calibración secundarias como pueden ser las ecuaciones de estado de sustancias simples como el óxido de magnesio (Speziale et al., 2001) o el oro (Dewaele et al., 2004), o la luminiscencia del rubí (Mao et al., 1986, Syassen, 2008). Este último método es el más extendido para medir la presión y está basado en el desplazamiento hacia 
mayores longitudes de onda que experimenta la banda R1 de la luminiscencia del rubí al aumentar la presión (figura 6).

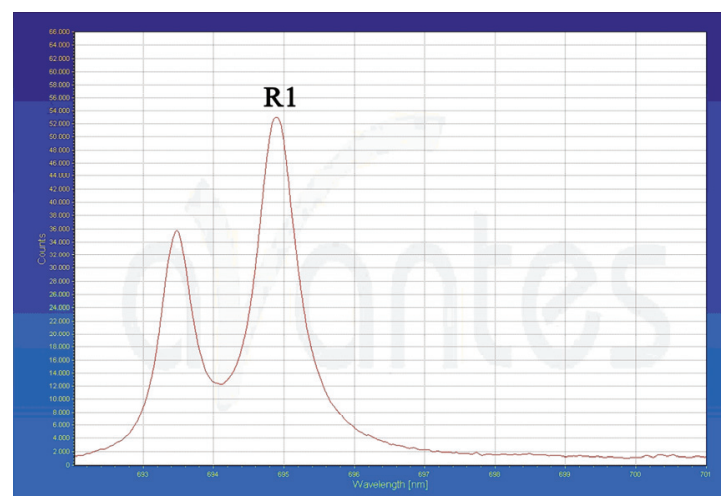

Fig. 6. Espectro de luminiscencia de un monocristal de rubí bajo condiciones hidrostáticas de presión (aproximadamente 1.6 GPa).

En las DACs las condiciones de alta presión se pueden combinar con las de alta temperatura, bien mediante calentamiento resistivo o bien mediante calentamiento láser. Esta última técnica es la única que permite reproducir las condiciones existentes en el interior terrestre en el laboratorio, aunque no está exenta de problemas pues se trata de experimentos tremendamente delicados. La técnica combinada se llama LHDAC (del íngles Laser-Heated Diamond-Anvil Cell) y consiste en calentar la muestra por medio de un láser infrarrojo de gran estabilidad y potencia por una o ambas caras de la celda. La desfocalización del láser minimiza el gradiente radial de temperaturas en la muestra bajo estudio y el calentamiento por ambos lados reduce significativamente el gradiente axial. Estos procedimentos persiguen obtener un calentamiento lo más homogeneo posible y, por consigiuente, conseguir un valor de la temperatura más preciso (Boehler, 2000).

¿Cómo sabemos la temperatura de nuestra muestra? Para determinar la temperatura en el interior de la celda se ajusta la ecuación de Planck a la radiación incandescente emitida por nuestra muestra:

$$
I(\lambda)=\frac{\varepsilon \cdot C_{1} \cdot \lambda^{-5}}{\left(e^{\left(C_{2} / \lambda \cdot T\right)}-1\right)}
$$

siendo $\mathrm{I}(\lambda)$ la intensidad de radiación emitida por la muestra, $\mathrm{C} 1$ y $\mathrm{C} 2$ constantes que dependen de constantes universales y $\varepsilon$ la emisividad de la muestra (Planck, 1914). En radiopirometría generalmente se supone que la emisividad es independiente de la longitud de onda en el rango de 400 a $900 \mathrm{~nm}$. Sin embargo, esta suposición y las imprecisiones experimentales pueden generar grandes incertidumbres en las temperaturas medidas (Benedetti \& Loubeyre, 2004). 
La técnica LHDAC ha permitido, por ejemplo, estudiar en profundidad los diagramas P-T de fases del hierro y sus aleaciones con elementos más ligeros y mejorar nuestro conocimiento del núcleo terrestre (Mao et al., 1990, Boehler, 1993, Zhang \& Guyot, 1999, Boehler et al., 2007, 2008, Errandonea et al., 2008, Santamaria-Perez \& Boehler, 2008). Para ello es preciso determinar la fase cristalina del hierro a esas presiones, su ecuación de estado y la curva de fusión. Pero la densidad calculada para el núcleo resulta menor que la del hierro elemental, por lo que se supone necesaria la existencia de otro elemento ligero que se disuelva en hierro fundido (núcleo externo) y forme aleaciones con el hierro sólido a alta presión (núcleo interno). La figura 7 muestra el diagrama de fases del hierro obtenida por Boehler y colaboradores (Boehler et al., 2008). Solo existen dos fases del hierro estables a altas presiones y altas temperaturas: una con estructura cúbica centrada en las caras (fcc) y otra hexagonal compacta (hcp). Por encima de 700.000 atmósferas, el hierro adquiere una estructura hcp y, por extrapolación de la curva de fusión hasta presiones de la interfase entre el núcleo externo e interno (330 GPa) se obtiene una temperatura próxima a los $5000^{\circ} \mathrm{C}$. Del conjunto de datos $\mathrm{P}-\mathrm{V}$ se obtuvo la ecuación de estado del metal y la compresibilidad de los ejes cristalográficos $a$ y $c$ de la celda unidad hexagonal. La relación $c / a$ del hierro hep decrece ligeramente con la presión, pero muestra un ligero aumento con la temperatura, por lo que en condiciones del núcleo tendría un valor similar al que posee a bajas presiones y temperaturas. Es importante destacar que, recientemente, Dubrovinsky y colaboradores han descubierto una fase cúbica centrada en el cuerpo (bcc) para la aleacción $\mathrm{Fe}_{0.9} \mathrm{Ni}_{0.1}$ estable por encima de $225 \mathrm{GPa}$ y $3100{ }^{\circ} \mathrm{C}$ (Dubrovinsky et al., 2007).

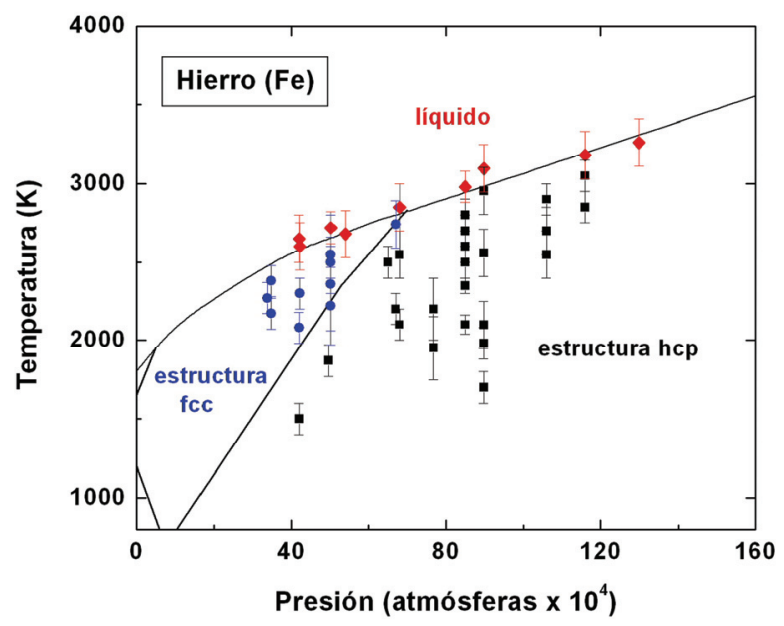

Fig. 7. Diagrama de fases del $\mathrm{Fe}$ a altas presiones y altas temperaturas obtenido usando la técnica de calentamiento láser en celda de diamante (LHDAC) y difracción de rayos X (Boehler et al., 2008). 


\section{Técnicas de caracterización a alta presión y alta temperatura}

\subsection{Radiación sincrotrón}

La combinación de las técnicas de alta presión y alta temperatura con las diferentes técnicas de caracterización derivadas de la radiación sincrotrón ha sido muy fructifera hasta la fecha para el campo de la Geofísica (Duffy, 2005), como veremos en adelante.

La radiación sincrotrón es la radiación electromagnética emitida por partículas cargadas al ser aceleradas o al ser desviadas de dirección. El electrón, debido a los cambios de dirección, emite radiación tangencialmente a su trayectoria circular. Esta radiación es aprovechada en grandes instalaciones llamadas precisamente sincrotrones para una gran variedad de aplicaciones (ver figura 8). En ellas, múltiples lineas tangenciales sirven la radiación sincrotrón a laboratorios que estudian los distintos fenómenos de interacción entre esta radiación y la materia.

La radiación sincrotrón supuso un gran avance tecnológico ya que trata de la fuente más poderosa de radiación en la región del ultravioleta y, sobretodo, de los rayos X. $\mathrm{Su}$ intensidad es muchos órdenes de magnitud mayor que los tubos ordinarios, es de altísima intensidad y además es sintonizable.

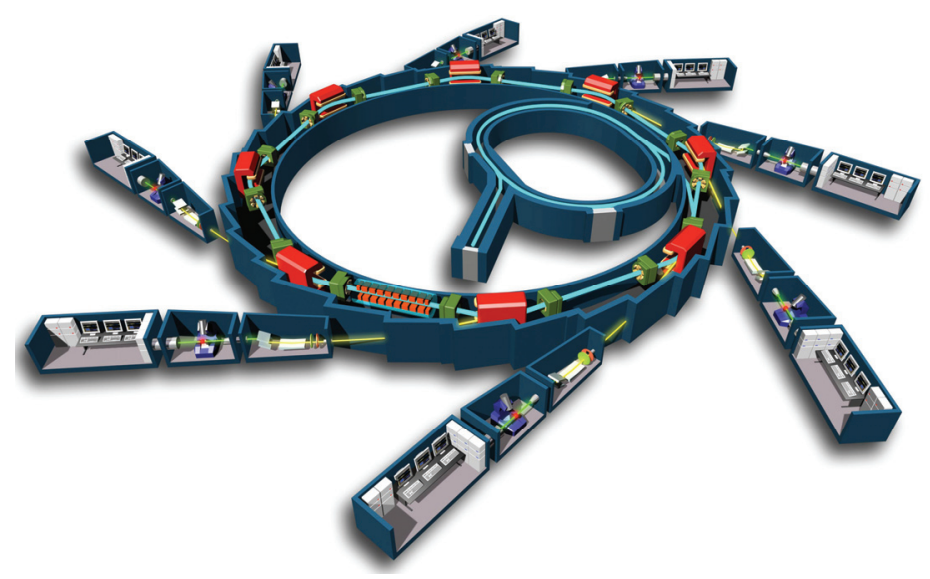

Fig. 8. Esquema que representa a un sincrotrón y las distintas estaciones o laboratorios que usan la radiación emitida tangencialmente.

Imagen: http://es.wikipedia.org/wiki/Sincrotr $\% \mathrm{C} 3 \% \mathrm{~B} 3 \mathrm{n}$

La interacción de esta radiación con la materia a estudiar produce distintos fenómenos: Una parte del haz de radiación incidente es absorbida por la muestra, otra es transmitida y otra dispersada. A partir de estos procesos se han desarrollado varias técnicas espectroscópicas, algunas de las cuales mencionaremos a continuación. 


\subsection{Difracción de rayos $X$}

La difracción es un fenómeno característico de las ondas que consiste en la dispersión de éstas cuando interaccionan con un objeto ordenado. Los rayos X tienen longitudes de onda similares a las distancias interatómicas en los materiales cristalinos, por lo que podemos usar la difracción de rayos X como método para estudiar el ordenamiento atómico. Del ángulo de difracción, a través de la ley de Bragg, se puede obtener las distancias características entre las distintas familias de planos cristalográficos y el tipo de celda unidad del material. De la intensidad de los picos de difracción se obtiene la disposición de los distintos átomos en la red periódica.

La difracción de rayos X permite la caracterización in situ de materiales de interés geofísico a altas presiones y altas temperaturas (Duffy, 2005, Schultz et al., 2005). Esta técnica se empleó con éxito, por ejemplo, para determinar la estructura de la fase post-perovskita del silicato de magnesio $(\mathrm{Mg}, \mathrm{Fe}) \mathrm{SiO}_{3}$ a $125 \mathrm{GPa}$ y 2500 K. En 2004, Murakami y colaboradores descubrieron que el mineral $(\mathrm{Mg}, \mathrm{Fe}) \mathrm{SiO}_{3}$, que en condiciones del manto inferior terrestre adopta la estructura perovskita, sufre una transición de fase en condiciones de presión y temperatura similares a las que se esperarían en la capa D" a una profundidad entre 2700 y $2900 \mathrm{~km}$ (Murakami et al., 2004, Oganov \& Ono, 2004). Esta nueva fase se denominó post-perovskita y se puede describir como una disposición laminar de octaedros $\left[\mathrm{SiO}_{6}\right]$ que comparten vértices a lo largo de un eje cristalográfico y aristas a lo largo de otro (vease la Figura 9). Este cambio estructural podría dar cuenta, al menos parcialmente, de la anisotropía de polarización de las ondas $\mathrm{S}$ y de la anticorrelación entre las velocidades $\mathrm{V}_{\mathrm{S}}$ y la velocidad del sonido $\mathrm{V}_{0}$. La discontinuidad sísmica de la capa $\mathrm{D}$ " sigue siendo objeto de debate con multiples vias de estudio que sugieren la importancia de los fenómenos de orientación preferencial y de las posibles heterogeneidades químicas en la explicación de las propiedades sísmicas.

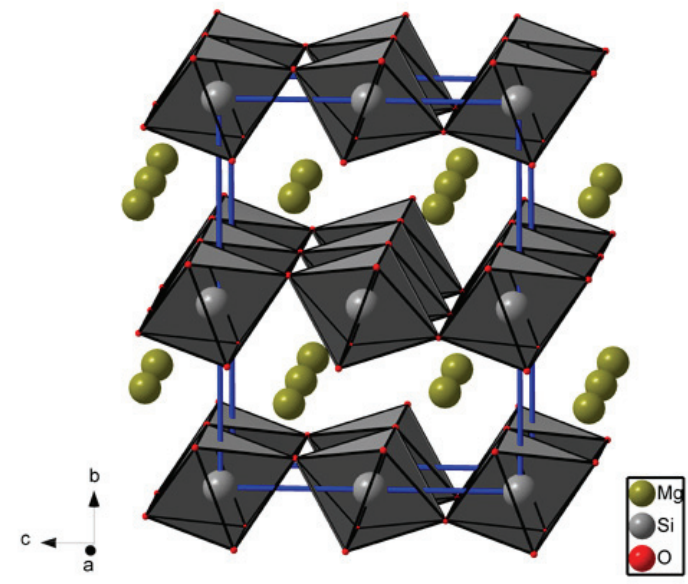

Fig. 9. Estructura de la fase post-perovskita del mineral $\mathrm{MgSiO}_{3}$. 
Otros estudios relevantes en condiciones extremas por difracción de rayos X son, por ejemplo, los realizados por Katsura y colaboradores (Katsura et al., 2003, Katsura et al., 2004) en una celda multi-yunque en los que se observaron la transición olivino-wadsleyita en $(\mathrm{Mg}, \mathrm{Fe})_{2} \mathrm{SiO}_{4}$ a $14.2 \mathrm{GPa}$ y $1600 \mathrm{~K}$ (discontinuidad a $410 \mathrm{~km}$ de profundidad) y la descomposicion de la wadsleyita en perovskita $\left(\mathrm{MgSiO}_{3}\right)$ y periclasa $(\mathrm{MgO})$ a $23 \mathrm{GPa}$ y $1900 \mathrm{~K}$ (discontinuidad de Repetti, $660 \mathrm{~km}$ de profundidad). También merecen especial consideración la determinación por rayos $\mathrm{X}$ de ecuaciones de estado de materiales de ínteres geofísico como pueden ser las aleaciones de hierro y elementos más ligeros (Mao et al., 1990, Zhang \& Guyot, 1999, Boehler et al., 2007, Errandonea et al., 2008, Santamaria-Perez \& Boehler, 2008, Santamaria-Perez et al., 2008) o de gases como el $\mathrm{H}_{2}$ en condiciones semejantes a las del interior de algunos planetas (Loubeyre et al., 1996).

\subsection{Absorción de rayos $X$}

Las técnicas radiográficas de rayos $\mathrm{X}$ (usando la absorción de los rayos $\mathrm{X}$ ), han sido empleadas para determinar la densidad de fluidos de interés planetario a altas presiones. En este método, una esfera de referencia es colocada en la muestra líquida e iluminada con rayos X monocromáticos. El contraste de la absorción entre la muestra y el material de la esfera puede usarse para determinar la densidad del fluido con un error menor al 1\%. Esta técnica ha permitido estudiar las densidades y viscosidades de líquidos del sistema Fe-FeS hasta una presión de 6 GPa y temperaturas por encima de los $1700 \mathrm{~K}$ (Sanloup et al., 2000, Sanloup et al., 2004). Se pudo comprobar cómo la adicción de azufre disminuye considerablemente la densidad y, consecuentemente, aumenta la compresibilidad del fundido.

Como se acaba de mencionar, la absorción de rayos $\mathrm{X}$ por la muestra dependerá de su composición química. El espectro de absorción de un material está determinado por su estructura electrónica y contiene diferentes umbrales de absorción debidos a las transiciones desde las capas electrónicas ocupadas más profundas a los niveles vacios. En sólidos y líquidos, para energías superiores a cada umbral se observan oscilaciones en el espectro que provienen de la interacción de los electrones excitados con los átomos vecinos. Asi pues, la espectroscopía de absorción de rayos $\mathrm{X}$ (XANES o EXAFS) nos proporciona información sobre el entorno local del átomo absorvente, sin ser necesario un orden atómico a largo alcance. Esto permite estudiar el entorno local de distintos cationes en una gran cantidad de minerales cristalinos, amorfos y fundidos. Varios estudios relevantes en muestras de ínteres geofísico empleando la técnica de absorción de rayos $\mathrm{X}$ han sido recogidos en el artículo de revisión monográfico de Brown y Parks (Brown \& Parks, 1989).

\subsection{Dispersión inelástica de rayos $X$}

En la investigación a alta presión, se han utilizado principalmente técnicas de dispersión elástica de rayos X (difracción) para el análisis estructural estático de las muestras de interés. Sin embargo, recientemente, la técnica sincrotrón de dispersión inelástica de rayos X está mostrando un enorme potencial en el estudio de la dinámica de materiales. En esta técnica, un fotón incidente de rayos $X$ con energía $E_{1}$ es 
absorvido por la muestra, la cual genera otro fotón de rayos $\mathrm{X}$ de energía diferente $\mathrm{E}_{2}$. El análisis de la energía y la dirección de la radiación $\mathrm{X}$ dispersada inelásticamente permite obtener las curvas de dispersión de las excitaciones elementales involucradas (dinámica electrónica y de fonones), a partir de las condiciones de conservación de la energía e impulso totales.

Esta técnica ha sido utilizada para medir la dispersión de los fonones acústicos longitudinales en la estructura hexagonal compacta (hcp) del hierro hasta $110 \mathrm{GPa}$ (figura 10). Fiquet y colaboradores obtuvieron las curvas de dispersión de fonones de una muestra de Fe policristalina que fue comprimida en una celda de diamante (Fiquet et al., 2001). Sus resultados revelan un aumento de la velocidad de propagación longitudinal de las ondas, $\mathrm{V}_{\mathrm{P}}$, con la presión. Los datos obtenidos siguen la ecuación de Birch para $V_{P}$, por lo que se extrapolaron las velocidades a las condiciones de presión del núcleo interno. La extrapolación de estos datos no concuerda con los resultados sísmicos, lo que lleva a pensar que el núcleo interno terrestre es entre un 4 y un $5 \%$ más ligero que el hierro hcp, por lo que debe contener elementos más ligeros. Estos resultados tuvieron una gran relevancia y fueron publicados en la revista Science en 2001.

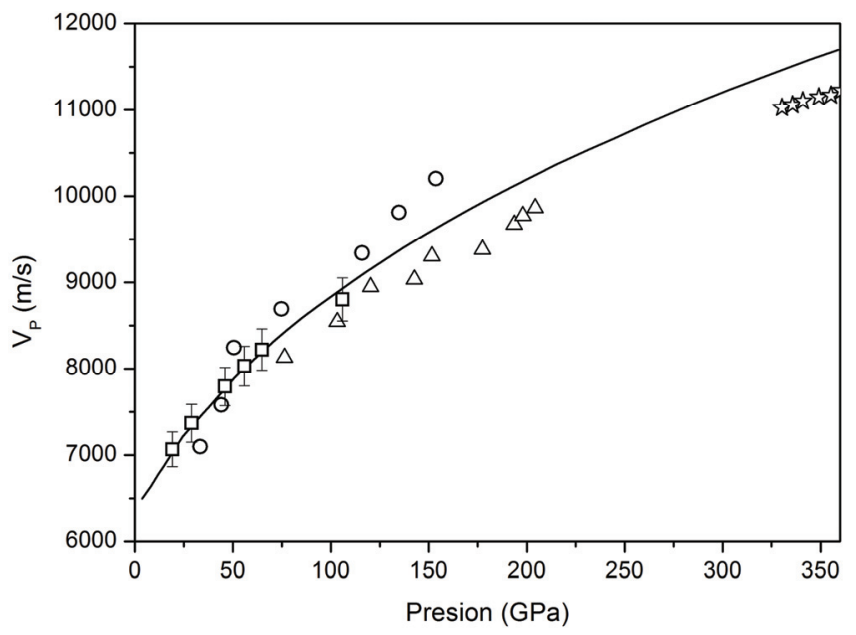

Fig. 10. Velocidad de propagación longitudinal de las ondas, $\mathrm{V}_{\mathrm{P}}$, en función de la presión. Los cuadrados, triángulos y círculos representan datos de dispersión inelástica de rayos $\mathrm{X}$ (Fiquet et al., 2001), de ondas de choque (Brown \& McQueen, 1986) y de dispersión inelástica resonante de rayos X (Mao et al., 2001), respectivamente. Las estrellas corresponden a datos sísmicos del núcleo interno (Dziewonski \& Anderson, 1981). La falta de acuerdo entre los datos del laboratorio y los sísmicos sugieren que el núcleo es aproximadamente un 5\% más ligero que el hierro hcp elemental. 


\section{Consorcio español de altas presiones: MALTA-Consolider Team}

Como hemos visto a lo largo de este capítulo, el estudio del comportamiento de la materia sometida a alta presión se ha consolidado como una línea de investigación esencial a nivel mundial.

Uno de los objetivos fundamentales de los estudios a alta presión es el de poder reproducir procesos como los que tienen lugar en el interior de la Tierra y de otros objetos planetarios (transformaciones de fase, reacciones químicas o formación de minerales e hidrocarburos, entre otros), y comprender sus implicaciones físicas, químicas y geológicas. Pero además, el desarrollo de esta disciplina científica trasciende el esclarecimiento de los procesos naturales, pues ha permitido desarrollar novedosas aplicaciones en diversas áreas tecnológicas, desde la ciencia de materiales hasta la tecnología de los alimentos.

La iniciativa Materia a Alta Presión (MALTA-Consolider) pretende incorporar a España al grupo de países líderes en la investigación a altas presiones, con el fin de que, a medio plazo, la sociedad española pueda beneficiarse de los avances derivados del conocimiento y control de esta singular tecnología de brillante presente y prometedor futuro. El proyecto MALTA-Consolider ha implementado las condiciones e infraestructura científica necesarias para llevar a cabo estudios interdisciplinares de los fenómenos mencionados e investigar sus aspectos fundamentales de manera coordinada. La sinergia de la cooperación entre los grupos participantes (ver figura 11) proporciona un valor añadido real al proyecto. La propuesta MALTA involucra actualmente a más de ochenta científicos de once grupos dedicados al estudio de fenómenos a altas presiones desde diferentes perspectivas. Esta iniciativa ha sido recientemente reforzada por la Comunidad de Madrid con la concesión del proyecto QUIMAPRES (Química a Alta Presión) y la integración de otros veinte científicos procedentes de grupos de investigación involucrados o interesados en las altas presiones.

Entre las infraestructuras con las que cuenta este consorcio destacan:

- Difractómetro de rayos X.

Permite determinar in situ la estructura cristalina de la muestra y su compresibilidad. Hasta la instalación de este equipo no existía en España ningún difractómetro capaz de operar a altas presiones.

- Espectrómetro de infrarrojo por transformada de Fourier.

Se trata de un equipo de altísima resolución y está modificado para acomodar celdas DAC y llevar a cabo experimentos de absorción en un amplísimo intervalo espectral.

- Espectrómetro Raman confocal.

Esta técnica es eficiente no solamente en materiales cristalinos sino también amorfos. El sistema permite realizar experimentos con diferentes excitaciones con la utilización de un láser de gas ( $\mathrm{Ar}-\mathrm{Kr}$ ionizados) y es ideal para llevar a cabo medidas en muestras micrométricas, como las contenidas en las DACs. 
- Prensa de gran volumen para sistemas líquidos.

Este equipo permite usar mayores volúmenes de muestra que en los sístemas de yunques opuestos y recuperar la muestra tras el tratamiento a altas presiones. Esto es partícularmente interesante en experimentos de síntesis y caracterización de hidratos y en muestras de interés biológico.

- Equipo de mecanizado.

Se dispone de un sistema de mecanizado para la fabricación y el montaje de dispositivos experimentales diseñados por los diferentes miembros del equipo.

- Cluster computacional para cálculos ab-initio.

Esta infraestructura permite simular fenómenos diversos de la materia a alta presión mediante cálculos computacionales de alto rendimiento.

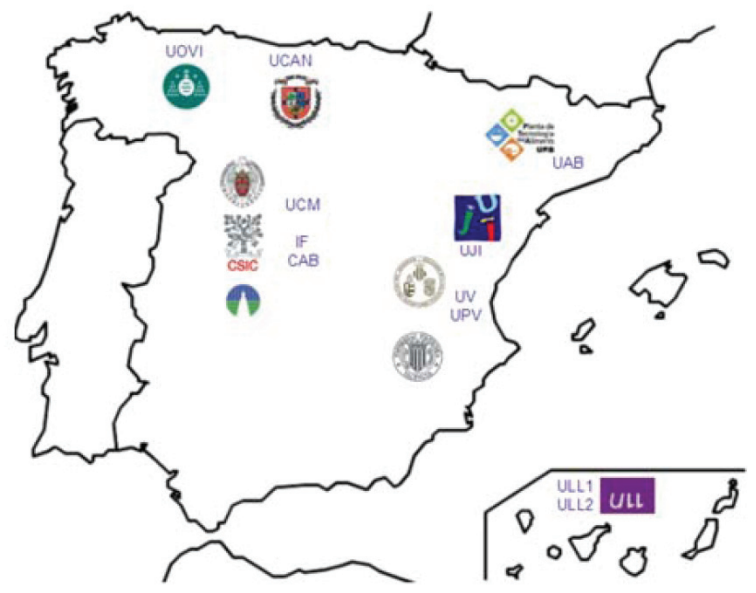

Fig.11. Instituciones que forman parte del consorcio MALTA-Materia a alta presión

El equipo MALTA-Consolider mantiene fructiferas colaboraciones con otros laboratorios de primer orden mundial, habiendo obtenido importantes resultados (www.malta-consolider.com) en estos tres años de funcionamiento y está siempre abierto a nuevas y prometedoras colaboraciones.

Como hemos visto, la experimentación a alta presión y alta temperatura ha resultado crucial para la comprensión de muchos fenómenos que ocurren en el interior de los planetas y muy probablemente lo seguirá siendo en un futuro próximo. Todos los estudios arriba mencionados ya han cambiado nuestro conocimiento del interior terrestre, aunque aún quedan muchos experimentos excitantes y novedosos por realizar. 


\section{Agradecimientos}

Este trabajo ha sido posible gracias al apoyo económico recibido tanto del Ministerio de Ciencia e Innovación Español (MICCIN) con los proyectos MALTAConsolider CSD2007-00045 y CTQ2009-14596-C02-01, como de la Comunidad de Madrid y el Fondo Social Europeo con el proyecto S2009/PPQ-1551 4161893 (QUIMAPRES).

\section{Referencias bibliográfcas}

BENEDETTI, L. R. \& LOUBEYRE, P. (2004). Temperature gradients, wavelengthdependent emissivity, and accuracy of high and very-high temperatures measured in the laser-heated diamond cell. High Pressure Res. 24, 423-445.

doi:10.1080/08957950412331331718; http://dx.doi.org/10.1080/08957950412331331718

BOEHLER, R. (1993). Temperatures in the Earth's core from melting-point measurements of iron at high static pressures. Nature 363, 534-536.

doi:10.1038/363534a0; http://dx.doi.org/10.1038/363534a0

BOEHLER, R. (2000). Laser heating in the diamond cell: techniques and applications. Hyperfine Interact. 128, 307-321. doi:10.1023/A:1012648019016; http://dx.doi.org/10.1023/A:1012648019016

BOEHLER, R., SANTAMARIA-PEREZ, D., ERRANDONEA, D. \& MEZOUAR, M. (2007). New experimental data on $\mathrm{Fe}$ and Fe-S melting and the temperature at the coremantle boundary. Geochim. Cosmochim. Acta 71, A103-A103.

BOEHLER, R., SANTAMARIA-PEREZ, D., ERRANDONEA, D. \& MEZOUAR, M. (2008). Melting, density, and anisotropy of iron at core conditions: new X-ray measurements to 150 GPa. J. Phys., Conf. Ser. 121, 022018.

doi:10.1088/1742-6596/121/2/022018; http://dx.doi.org/10.1088/1742-6596/121/2/022018

BROWN, G. E. J. \& PARKS, G. A. (1989). Synchrotron-based X-ray absorption studies of cation environments in Earth materials. Reviews of Geophysics 27, 519-533. doi:10.1029/RG027i004p00519; http://dx.doi.org/10.1029/RG027i004p00519

BROWN, J. M. \& MCQUEEN, R. G. (1986). Phase-transitions, Gruneisen-parameter, and elasticity for shocked iron between 77 and 400 GPa. Journal of Geophysical ResearchSolid Earth and Planets 91, 7485-7494. doi:10.1029/JB091iB07p07485; http://dx.doi.org/10.1029/JB091iB07p07485

ITO, E. \& FUNAKOSHI, K. (2004). Olivine-wadsleyite transition in the system $(\mathrm{Mg}, \mathrm{Fe})_{2} \mathrm{SiO}_{4}$. J. Geophys. Res.-Solid Earth 109, 12.

KATSURA, T., YAMADA, H., SHINMEI, T., KUBO, A., ONO, S., KANZAKI, M., YONEDA, A., WALTER, M. J., ITO, E., URAKAWA, S., FUNAKOSHI, K. \& UTSUMI, W. (2003). Post-spinel transition in $\mathrm{Mg}_{2} \mathrm{SiO}_{4}$ determined by high P-T in situ X-ray diffractometry. Phys. Earth Planet. Inter. 136, 11-24. 
doi:10.1016/S0031-9201(03)00019-0;

http://dx.doi.org/10.1016/S0031-9201(03)00019-0

LOUBEYRE, P., LETOULLEC, R., HAUSERMANN, D., HANFLAND, M., HEMLEY, R. J., MAO, H. K. \& FINGER, L. W. (1996). X-ray diffraction and equation of state of hydrogen at megabar pressures. Nature 383, 702-704.

doi:10.1038/383702a0;

http://dx.doi.org/10.1038/383702a0

MAO, H. K., WU, Y., CHEN, L. C., SHU, J. F. \& JEPHCOAT, A. P. (1990). Static compression of iron to $300 \mathrm{GPa}$ and $\mathrm{Fe}_{0.8} \mathrm{Ni}_{0.2}$ alloy to $260 \mathrm{GPa}$ : Implications for composition of the core. Journal of Geophysical Research-Solid Earth and Planets 95, 21737-21742. doi:10.1029/JB095iB13p21737; http://dx.doi.org/10.1029/JB095iB13p21737

MAO, H. K., XU, J. \& BELL, P. M. (1986). Calibration of the ruby pressure gauge to 800 Kbar under quasi-hydrostatic conditions. Journal of Geophysical Research-Solid Earth and Planets 91, 4673-4676. doi:10.1029/JB091iB05p04673; http://dx.doi.org/10.1029/JB091iB05p04673

MAO, H. K., XU, J., STRUZHKIN, V. V., SHU, J., HEMLEY, R. J., STURHAHN, W., HU, M. Y., ALP, E. E., VOCADLO, L., ALFE, D., PRICE, G. D., GILlAN, M. J., SCHWOERER-BOHNING, M., HAUSERMANN, D., ENG, P., SHEN, G., GIEFERS, H., LUBBERS, R. \& WORTMANN, G. (2001). Phonon density of states of iron up to 153 gigapascals. Science 292, 914-916.

doi:10.1126/science.1057670;

http://dx.doi.org/10.1126/science. 1057670

MURAKAMI, M., HIROSE, K., KAWAMURA, K., SATA, N. \& OHISHI, Y. (2004). Post-perovskite phase transition in $\mathrm{MgSiO}_{3}$. Science 304, 855-858.

doi:10.1126/science.1095932;

http://dx.doi.org/10.1126/science.1095932

NELLIS, W. J. (2006). Dynamic compression of materials: metallization of fluid hydrogen at high pressures. Rep. Prog. Phys. 69, 1479-1580.

doi:10.1088/0034-4885/69/5/R05; http://dx.doi.org/10.1088/0034-4885/69/5/R05

NELLIS, W. J., WEIR, S. T. \& MITCHELL, A. C. (1996). Metallization and electrical conductivity of hydrogen in Jupiter. Science 273, 936-938.

doi:10.1126/science.273.5277.936;

http://dx.doi.org/10.1126/science.273.5277.936

OGANOV, A. R. \& ONO, S. (2004). Theoretical and experimental evidence for a postperovskite phase of $\mathrm{MgSiO}_{3}$ in Earth's D" layer. Nature 430, 445-448.

doi:10.1038/nature02701;

http://dx.doi.org/10.1038/nature02701

PLANCK, M. (1914). The theory of heat radiation, Second edition ed. Philadelphia: Blackiston's Son \& Co.

SANLOUP, C., FIQUET, G., GREGORYANZ, E., MORARD, G. \& MEZOUAR, M. (2004). Effect of Si on liquid Fe compressibility: Implications for sound velocity in core materials. Geophys. Res. Lett. 31, 4. 
doi:10.1029/2004GL019526;

http://dx.doi.org/10.1029/2004GL019526

SANLOUP, C., GUYOT, F., GILLET, P., FIQUET, G., MEZOUAR, M. \& MARTINEZ, I. (2000). Density measurements of liquid Fe-S alloys at high-pressure. Geophys. Res. Lett. $27,811-814$. doi:10.1029/1999GL008431; http://dx.doi.org/10.1029/1999GL008431

SANTAMARIA-PEREZ, D. \& BOEHLER, R. (2008). FeSi melting curve up to $70 \mathrm{GPa}$. Earth Planet. Sci. Lett. 265, 743-747. doi:10.1016/j.eps1.2007.11.008; http://dx.doi.org/10.1016/j.epsl.2007.11.008

SANTAMARIA-PEREZ, D., ERRANDONEA, D., VEGAS, A., NUSS, J., JANSEN, M., RODRIGUEZ-HERNANDEZ, P., MUNOZ, A. \& BOEHLER, R. (2008). Phase diagram studies on iron and nickel silicides: high-pressure experiments and ab initio calculations. J. Phys., Conf. Ser. 121, 022013. doi:10.1088/1742-6596/121/2/022013; http://dx.doi.org/10.1088/1742-6596/121/2/022013

SCHULTZ, E., MEZOUAR, M., CRICHTON, W., BAUCHAU, S., BLATTMANN, G., ANDRAULT, D., FIQUET, G., BOEHLER, R., RAMBERT, N., SITAUD, B. \& LOUBEYRE, P. (2005). Double-sided laser heating system for in situ high-pressure high-temperature monochromatic X-ray diffraction at the ESRF. High Pressure Res. 25, 71-83.

doi:10.1080/08957950500076031; http://dx.doi.org/10.1080/08957950500076031

SPEZIALE, S., ZHA, C. S., DUFFY, T. S., HEMLEY, R. J. \& MAO, H. K. (2001). Quasihydrostatic compression of magnesium oxide to $52 \mathrm{GPa}$ : Implications for the pressurevolume-temperature equation of state. J. Geophys. Res.-Solid Earth 106, 515-528. doi:10.1029/2000JB900318; http://dx.doi.org/10.1029/2000JB900318

SYASSEN, K. (2008). Ruby under pressure. High Pressure Res. 28, 75-126. doi:10.1080/08957950802235640; http://dx.doi.org/10.1080/08957950802235640

WEIR, S. T., MITCHELL, A. C. \& NELLIS, W. J. (1996). Metallization of fluid molecular hydrogen at 140 GPa. Phys. Rev. Lett. 76, 1860-1863. doi:10.1103/PhysRevLett.76.1860; http://dx.doi.org/10.1103/PhysRevLett.76.1860

WIGNER, E. \& HUNTINGTON, H. B. (1935). On the possibility of a metallic modification of hydrogen. J. Chem. Phys. 3, 764-770.

doi:10.1063/1.1749590; http://dx.doi.org/10.1063/1.1749590

ZHANG, J. \& GUYOT, F. (1999). Thermal equation of state of iron and $\mathrm{Fe}_{0.91} \mathrm{Si}_{0.09}$. Phys. Chem. Miner. 26, 206-211.

doi:10.1007/s002690050178;

http://dx.doi.org/10.1007/s002690050178 